\title{
Effets de la granulométrie et de l'humidité sur la palatabilité des aliments offerts aux chèvres
}

\author{
P Morand-Fehr, J Hervieu, T Ouedraogo
}

\begin{abstract}
INRA, station de nutrition et alimentation de I'INA-PG, 16. rue Claude-Bernard, 75231 Paris cedex 05, France
\end{abstract}

La palatabilité de 2 aliments, une orge $(O)$ et des pulpes de betteraves deshydratées (P), a été estimée à différentes granulométries et humidités grâce aux tests mis au point sur aliments secs et humides (Morand-Fehr et al, 1987, 1992). Deux aliments placés dans 4 coupelles sont présentés à 14 chèvres, individuellement, au cours d'une séquence $4 \times 30 \mathrm{~s}$. Trois tailles de particules : passant au tamis de $1 \mathrm{~mm}(F)$, de $5 \mathrm{~mm}(M)$ et ne passant pas au tamis de $5 \mathrm{~mm}(\mathrm{G})$, et 3 taux d'humidité : $10 \%(\mathrm{~S}), 40 \%\left(\mathrm{H}_{1}\right)$ et $70 \%\left(\mathrm{H}_{2}\right)$ sont testés. Les aliments $\mathrm{H}_{1}$ et $\mathrm{H}_{2}$ sont broyés à sec et humidifiés à 40 et $70 \%$ avant d'être soumis au tamisage. Pour $O$ et $P$, on a comparé les 3 taux d'humidité à même granulométrie et les 3 granulométries à même humidité, excepté la granulométrie $\mathrm{F}$ pour $\mathrm{O}$ en raison de l'obtention d'un aliment pâteux. Dans chaque cas, 3 aliments sont comparés 2 à 2 dans un dispositif de carré latin.

À même humidité, la palatabilité des aliments à granulométrie grossière (G) apparaî́t significativement supérieure à celle des aliments de granulométrie $M$, alors que les aliments $F$ ( $P$ ou $O$ ) sont quasiment refusés. À même granulométrie, les diverses humidités ont des effets différents pour $O$ et $P$. La matière brute $(M B)$ ingérée de $P$ augmente avec le taux d'humidité alors que la MS ne varie pas significativement. La MB de $O$ augmente légèrement puis diminue lorsque la capacité d'absorption d'eau est atteinte (cas de l'orge à $70 \%$ d'humidité) alors que la MS diminue nettement quand l'humidité s'élève mais l'amélioration de la palatabilité due au taux d'humidité semble sensiblement plus importante dans le cas d'aliment à granulométrie fine que grossière (g MS): PFS : $4,3(8,3), \mathrm{PFH}_{2}: 50,3(24,3)$, PGS: 110,6 (77,0), PGH2: 65,3 (22,7).

Les effets de la granulométrie apparaissent importants mais peu liés à la nature de l'aliment alors que ceux de l'humidité dépendraient de leur pouvoir de rétention d'eau lié à la nature de leur fraction glucidique.

Morand-Fehr P, Hervieu J, Legendre D, Gutter A, Del Tedesco L (1987) Ann Zootech 36, 324 Morand-Fehr P. Hervieu J (1993) Ann Zootech 42, 192

Tableau I. Quantités moyennes d'aliments ingérés pendant les tests.

\begin{tabular}{|c|c|c|c|c|c|}
\hline \multicolumn{3}{|c|}{ Pulpes de betteraves } & \multicolumn{3}{|c|}{ Orge } \\
\hline Aliment & $M S(g)$ & $M B(g)$ & Aliment & $M S(g)$ & $M B(g)$ \\
\hline $\begin{array}{c}\text { Comparal } \\
\text { PF } \\
\text { PM } \\
\text { PG }\end{array}$ & $\begin{array}{r}7 \text { des aliments } \\
0,9^{\mathrm{a}}(1,8) \\
70,6^{\mathrm{b}}(41,6) \\
113,2^{\mathrm{c}}(49,5)\end{array}$ & $\begin{array}{r}\text { Sme humidité } \\
2,1^{\mathrm{a}}(4,1) \\
144,2^{\mathrm{b}}(69,4) \\
211,3^{\mathrm{c}}(78,7)\end{array}$ & $\begin{array}{l}O M \\
O G\end{array}$ & $\begin{array}{l}107,4^{a}(40,5) \\
148,9^{b}(49,5)\end{array}$ & $\begin{array}{ll}179,0^{\mathrm{a}} & (67,4) \\
248,1^{\mathrm{b}} & (82,5)\end{array}$ \\
\hline $\begin{array}{c}\text { Comparal } \\
\mathrm{PS} \\
\mathrm{PH}_{1} \\
\mathrm{PH}_{2}\end{array}$ & $\begin{array}{c}\text { des aliments } \\
55,8^{\mathrm{a}}(43,6) \\
70,8^{\mathrm{a}}(32,7) \\
60,4^{\mathrm{a}}(22,2)\end{array}$ & $\begin{array}{r}\text { ame granulomét } \\
62,0^{\mathrm{a}}(49,3) \\
118,0^{\mathrm{b}}(54,5) \\
201,4^{\mathrm{c}}(73,8)\end{array}$ & $\begin{array}{l}\mathrm{OS} \\
\mathrm{OH}_{1} \\
\mathrm{OH}_{2}\end{array}$ & $\begin{array}{r}187,7^{\mathrm{a}}(66,6) \\
147,3^{\mathrm{a}}(44,1) \\
55,0^{\mathrm{b}}(31,8)\end{array}$ & $\begin{array}{ll}208,6^{\mathrm{a}} & (74,0) \\
245,5^{\mathrm{b}} & (73,5) \\
183,4^{\mathrm{a}} & (106,1)\end{array}$ \\
\hline
\end{tabular}

Les résultats sur une colonne n'ayant pas de lettres communes $\left({ }^{a}, b^{b}\right.$ ou $\left.c\right)$ sont significativement différents $(P<0.05)$. 